\title{
TOO CLOSE FOR COMFORT: EVALUATING A REWARD-BASED APPROACH TO INCREASE DRIVERS' HEADWAY
}

\author{
Robert C. Ramkhalawansingh ${ }^{1}$ \& Lana M. Trick ${ }^{2}$ \\ ${ }^{1}$ University of Toronto, Toronto, Ontario, Canada \\ ${ }^{2}$ University of Guelph, Guelph, Ontario, Canada \\ E-mails: robert.ramkhalawansingh@uhn.ca and ltrick@uoguelph.ca
}

\begin{abstract}
Summary: Tailgating is often implicated as a leading contributor to rear-end collisions but this behaviour is difficult to remediate because drivers are poor at estimating their own headway. Our first goal was to compare novice and fully licensed drivers as they applied existing headway interventions in a driving simulator. Our second goal was to develop an automated, reward-based approach to encourage longer headways. We first compared headway in the driving simulator to previous studies on real-world car following behaviour by asking drivers to (i) achieve what they perceived to be the minimum safe headway or to (ii) employ the common "2 second rule" intervention. We observed a close agreement between the headways achieved in the simulator and those achieved in prior real-world car-following paradigms. We then implemented our headway evaluation system and compared headway across instruction type: (i) minimum safe headway, (ii) "2 second rule", or (iii) the headway evaluation system. We observed that fully licensed motorists maintained the longest headways while using our system. While drivers reported that the headway evaluation system was easy and appealing to use, they did not foresee continuing to use the device in the future. The current system may be beneficial for driver training applications or to promote situation awareness during the use of automated driver assistance systems such as adaptive cruise control.
\end{abstract}

\section{INTRODUCTION}

Rear-end collisions account for up to $30 \%$ of all car accidents and driver tailgating is a noteworthy contributor (Wang et al., 2011). It can take 2.5 seconds or greater for a driver to respond to a forward hazard but motorists routinely tailgate by maintaining headways of 1 second or less (Wang \& Song, 2009; Michael et al., 2000). Interestingly, while many driving skills improve with experience, drivers also reduce their safety margin. It has been demonstrated that headway decreases with increased driving experience, possibly because drivers learn that they can follow at a short headway yet avoid negative consequences (Duncan et al., 1991). The goal of the current study was to investigate the efficacy of a novel tailgating intervention that relies on an automated feedback system to shape car-following behaviour through reward. To accomplish this goal we used a driving simulator because of the inherent danger in measuring tailgating on real roads. This paper presents the results of two experiments: the first experiment was designed to validate the driving simulator by comparing the headway that drivers maintain in the simulator to earlier studies on headway during real driving. The second experiment was designed to investigate the impact of a novel, reward-based system for promoting appropriate following distance. 


\section{EXPERIMENT 1}

Driving simulators are the safest way to measure dangerous driving behaviours such as tailgating. However, it has been suggested that drivers underestimate their headway in driving simulators (Panerai et al., 2001). Thus our first goal was to validate the driving simulator by observing the headways that drivers attained in the simulator while employing a common tool for teaching appropriate following distances: the " 2 second rule". Because drivers are typically poor at estimating their own headway (Taieb-Maimon, 2007), many places in North America, including New York and Ontario, teach motorists to use the 2 second rule to estimate headway. This heuristic requires drivers to count the number of seconds between a lead vehicle passing a given landmark (e.g. post) and the driver's vehicle passing that same landmark. Taieb-Maimon (2007) conducted a study on a road course where drivers were instructed to use a counting technique akin to the 2 second rule and established that drivers overestimate their headway. Whether this headway overestimation is prevalent in a driving simulator remains unclear. Our second goal was to compare drivers by level of licensure, given that tailgating worsens with experience (e.g. Duncan et al., 1991). Therefore we compared novice and fully licensed, young drivers. The novice drivers held their G1 license in Ontario's graduated licensing system, which is obtained after a written exam and mandates the supervision of a fully licensed driver. The more experienced group held an Ontario G license or a full-privilege driver's license. We predicted that $G$ licensed drivers would maintain shorter headways than the G1 drivers.

\section{METHOD}

\section{Participants}

48 young adults between the ages of 18 and $24(M=20, S D=2.04)$ from the University of Guelph participant pool took part in the experiment. 24 held an Ontario G license and 24 held a G1 license (see Table 1 for drive characteristics). A total of 53 participants were recruited but 3 G1 licensed drivers and $2 \mathrm{G}$ licensed drivers withdrew due to simulator sickness.

Table 1. Summary of participant characteristics for experiment 1

\begin{tabular}{lcccccc}
\hline Driver Group & $n$ & Mean Age $(S D)$ & $\begin{array}{c}\text { Mean Months } \\
\text { Licensed }(S D)\end{array}$ & $\begin{array}{c}\text { Mean Km per } \\
\text { day }(S D)\end{array}$ & $\begin{array}{c}\text { Driver } \\
\text { accidents }\end{array}$ & $\begin{array}{c}\text { Frequency of } \\
\text { Men/Women }\end{array}$ \\
$\begin{array}{c}\text { Full License } \\
\text { 2 Second Rule }\end{array}$ & 12 & $20.83(1.69)$ & $55.33(16.84)$ & $53.16(32.63)$ & 2 & $10 / 2$ \\
$\quad \begin{array}{c}\text { Minumum Safe } \\
\text { Learning Drivers }\end{array}$ & 12 & $22.25(2.13)$ & $61.50(22.61)$ & $71.82(69.15)$ & 5 & $10 / 2$ \\
2 Second Rule & 12 & $19.25(1.35)$ & $12.08(7.13)$ & $17.92(24.69)$ & 6 & $7 / 5$ \\
Minimum Safe & 12 & $18.5(0.67)$ & $14.67(6.93)$ & $12(14.07)$ & 3 & $2 / 10$ \\
\hline
\end{tabular}

\section{Apparatus and Stimuli}

Testing was carried out in a fixed-base simulator that consisted of a full car body surrounded by 300 degree wrap-around screens (Drive Safety DS600c, equipped with force feedback steering, vibration transduction, and a 5.1 channel sound system). The tailgating paradigm consisted of a straight, two-lane rural highway with light oncoming traffic. A lead vehicle was programmed to 
traverse the route at a rate of $80 \mathrm{~km} / \mathrm{h}$ but to gently vary in speed between $75 \mathrm{~km} / \mathrm{h}$ and $80 \mathrm{~km} / \mathrm{h}$ at a maximum rate of $0.5 \mathrm{~m} / \mathrm{s} / \mathrm{s}$ to make it necessary for the driver to actively adjust their headway. The simulation always began with the lead vehicle waiting at a four-way stop and the driver positioned several meters behind the lead vehicle. The lead vehicle would gradually accelerate from 0 to $80 \mathrm{~km} / \mathrm{h}$ after the driver approached the rear of the lead vehicle.

\section{Procedure}

Participants were randomly assigned to one of two types of headway instructions: drivers in the minimum safe headway group were instructed to follow at the closest headway that they could maintain from the lead vehicle that would enable them to stop in time if the lead driver were to suddenly brake (Taieb-Maimon \& Shinar, 2001). Drivers assigned to the 2 second rule group were provided with the instructions outlined by the Ontario Ministry of transportation: (i) pick a marker on the roadside (e.g. a pole), (ii) when the lead vehicle passes the marker, start counting "one thousand and one, one thousand and two", (iii) stop counting when your vehicle reaches the marker. Participants in both headway instruction groups were told that the lead vehicle was being driven by a friend, whom they were to safely follow to their destination. After a 6 minute practice drive, participants embarked on two 16 minute experimental drives.

\section{RESULTS AND DISCUSSION}

Time headway was measured as a function of headway instruction type ( 2 second rule, minimum safe distance) and licensure (G1, G license) using mixed factorial ANOVAs. Our principle goal was to validate the simulator for use in later headway studies. As expected, the measures taken in our simulator were similar to those observed in previous real-world car following studies. Fully licensed drivers in the minimum safe headway condition achieved headways similar to those observed by Otha (1994, as cited by Taieb- Maimon \& Shinar, 2001) where drivers were asked to maintain a comfortable distance. Similarly, fully licensed drivers employing the 2 second rule compared closely with drivers using a similar counting technique employed by Taieb-Maimon (2007), see Figure 1. Thus the simulator appeared to provide a suitable paradigm in which to study tailgating. Our second goal was to examine differences in headway as a function of headway instruction and licensure. We observed that headway instruction had no effect on headway or standard deviation in headway. The 2-second rule was no more effective than the minimum safe headway instructions at encouraging drivers to maintain longer headways. However, as predicted a main effect of driving experience on driver headway emerged, $F(1,41)=7.53, p=.009$, Partial $\eta^{2}=.16$, in which fully licensed drivers demonstrated their characteristic headway overestimation (see Figure 2).

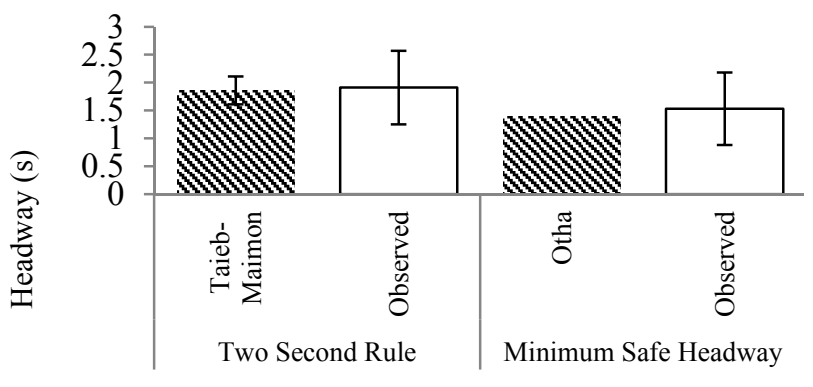

Figure 1. Headway in the simulator compared to previous on-road investigations

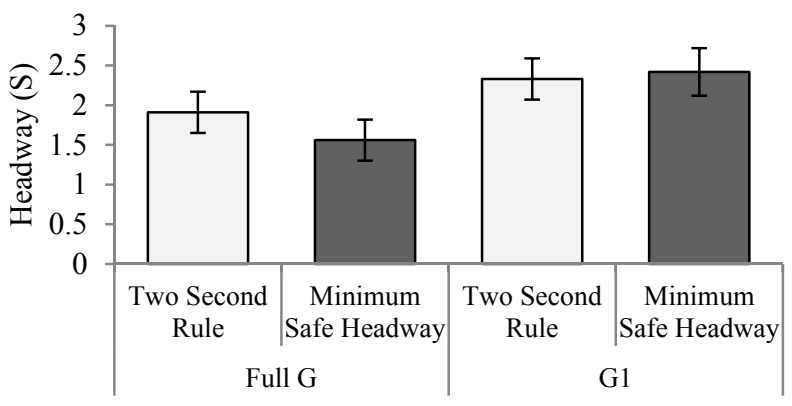

Figure 2. Headway by instruction type and level of licensure 


\section{EXPERIMENT 2}

The goal of this experiment was to investigate a novel, reward-based approach to address the antecedents of tailgating. Drivers are poor at estimating their own headway and even when applying the 2 second rule intervention, fully licensed drivers overestimate their headway. Our approach aims to facilitate more accurate headway estimation. Furthermore, tailgating worsens with experience, as evidenced by the differences in headway between our novice and fully licensed drivers. This may be due to a lack of feedback pertaining to headway performance and thus we endeavored to use positive feedback to encourage longer headways. While automakers have been implementing adaptive cruise control and collision avoidance systems that can initiate braking in response to an impending front to rear-end collision, these systems fail to address the factors that give rise to tailgating. Automation can also diminish the driver's capacity to thwart potential collisions independently by undermining situation awareness, limiting skill acquisition, and promoting distraction (Lee, 2007). Instead, we propose to convey headway information to the driver using an in-vehicle display called a headway evaluation system. The system is designed to provide the driver with (i) an objective measure of headway in real-time along with (ii) a long-term representation of performance in the form of tokens that one collects as reinforcement for safe headways of 2 seconds and beyond. Our first goal was to compare performance across two versions of the headway evaluation system: (i) the real-time gauge only and (ii) the real-time gauge with the long-term gauge present. We predicted that headway would be greatest in the presence of the long-term headway gauge. Our second goal was to compare headway in the presence of the headway evaluation system to the headways that we observed in Experiment 1 where drivers maintained either the 2 second rule or a minimum safe headway. We hypothesized that headway would be greater while using the headway evaluation system. To assess any potential negative effects of viewing the display while driving, we also captured mental workload using the NASA-TLX and standard deviation in lateral position.

\section{METHODS}

\section{Participants}

Twenty fully licensed drivers, 10 female and 10 male, aged of 18 to $23(M=19.5, S D=1.57)$, were recruited from the University of Guelph participant pool. Mean length of licensure was 37.4 months (see Table 2 for summary of participant characteristics).

Table 2. Summary of participant characteristics for experiment 2

\begin{tabular}{ccccccc}
\hline $\begin{array}{c}\text { Experience } \\
\text { Level }\end{array}$ & $n$ & $\begin{array}{c}\text { Mean Age } \\
(S D)\end{array}$ & $\begin{array}{c}\text { Frequency } \\
\text { Men/Women }\end{array}$ & $\begin{array}{c}\text { Mean Months } \\
\text { Licensed }(S D)\end{array}$ & $\begin{array}{c}\text { Mean Estimated Km } \\
\text { driven per day }(S D)\end{array}$ & $\begin{array}{c}\text { Accident- } \\
\text { Involved } \\
\text { Drivers }\end{array}$ \\
\hline Full G & 20 & $19.5(1.57)$ & $10 / 10$ & $37.45(17.90)$ & $54.05(77.11)$ & 7 \\
\hline
\end{tabular}

\section{Stimulus and Apparatus}

The testing employed the same apparatus as Experiment 1 but with the addition of a 7" Lilliput LCD monitor with a native resolution of $640 \times 480$ pixels to display the headway evaluation system (see Figure 3). The real-time component of the display reported headway(s) based on the position 
of a needle along a vertical scale and distance (meters) in the head of the needle. The needle was colored green when headway was safe ( $\geq 2$ seconds), turning red at $<2$ s (see Figure $4 a$ ). The longterm component of the display awarded a green token for every 10 s period in which headway was $\geq 2$ s. The rightmost token represents the most recent 10 seconds of driving. If headway was $\leq 2$, a token would be withheld for the ten second period in which tailgating occurred (see Figure $4 \mathrm{~b}$ ).

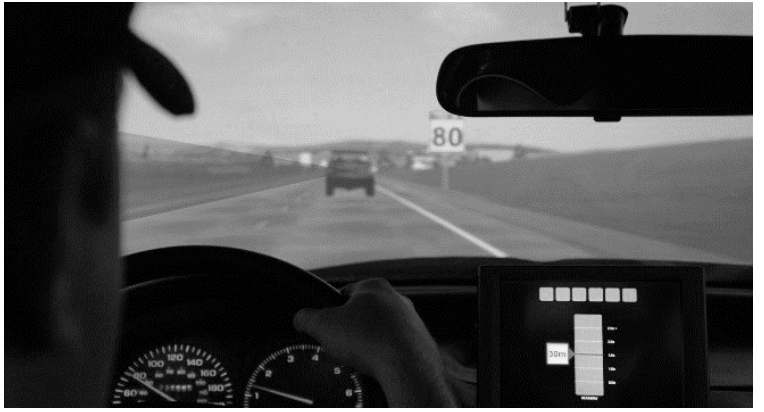

Figure 3. Depiction of the headway evaluation system inside the simulator

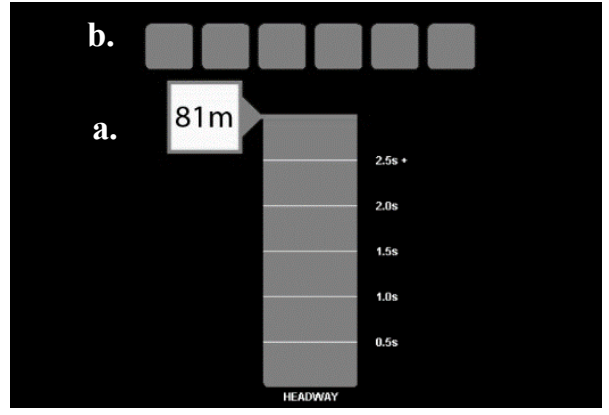

Figure 4. Depiction of the (a) real-time and (b) long-term headway gauges

\section{PROCEDURE}

During a six minute practice session, the experimenter explained to drivers how the real-time and long-term components of the headway evaluation system worked. The drivers were then asked to complete the same driving task outlined in Experiment 1. An interview was conducted after the simulation to assess how participants felt about the appeal, ease of use, and efficacy of the system using a series of 7-point likert scales. The NASA-TLX was also administered at this time.

\section{RESULTS AND DISCUSSION}

We employed a within-subjects design in which drivers experienced two versions of the headway evaluation system: (i) real-time headway gauge only or (ii) real-time gauge + long-term gauge. The order in which the drivers experienced the different versions of the system was counterbalanced. Contrary to our hypothesis, the headway that drivers maintained while using the long-term gauge was not significantly greater than the headway that they achieved while relying on real-time feedback alone, $t(18)=.495, p=.627$. While the inclusion of the long-term gauge did not increase time headway relative to the real-time gauge alone, it decreased standard deviation in lateral position, $t(18)=1.83, p=.084, d=.445$, possibly because of its low visual eccentricity from the driver's normal line of sight (Wittmann et al., 2006). Our second goal was to compare headway across instruction type: (i) Minimum Safe Headway, (ii) 2 second rule, and (iii) the headway evaluation system with both gauges active. There was a significant effect of headway instruction type on time headway(s), $F(2,40)=13.57, p<.001$, partial $\eta^{2}=.40$. Post hoc comparisons revealed that motorists maintained significantly greater headways when using the headway evaluation system than they did when following the instruction to maintain a minimum safe headway or while employing the 2 second rule, see Figure 5. The headway evaluation system promoted a mean headway of 2.58 seconds with both gauges present, a headway that may be more conducive to motorists recognizing and averting a potential collision in the event of rapid lead vehicle deceleration. 


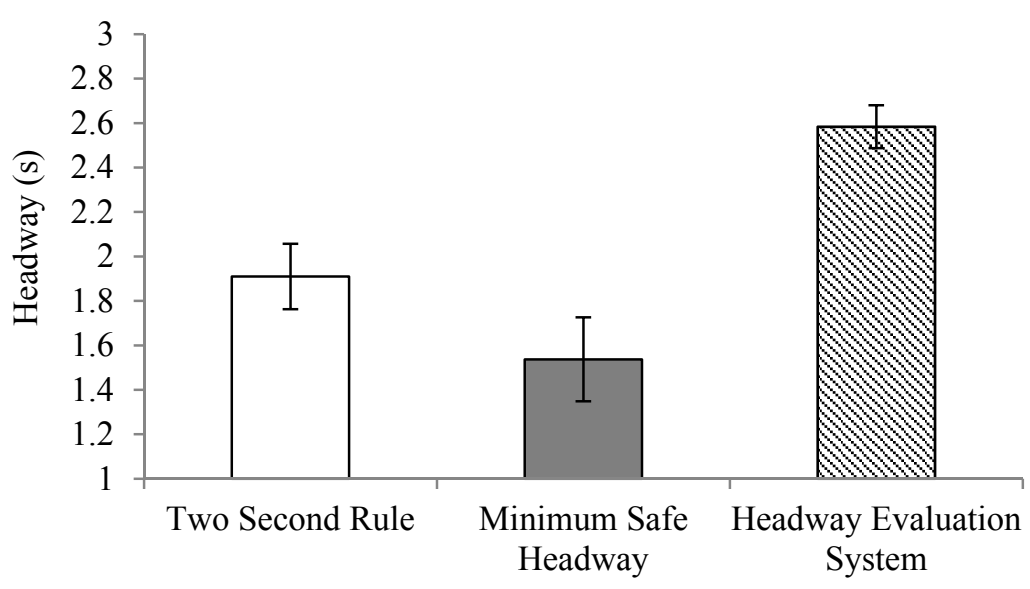

Figure 5. Driver headway (s) by headway intervention type

While the headway evaluation system promoted longer headways, drivers reported that the system required a higher level of mental workload to use, as measured by the NASA-TLX, than the 2 second rule or the minimum safe headway instructions, $F(2,40)=5.57, p=.007$, Partial $\eta^{2}=.064$. However, the workload score $(M=35$ out of 100$)$ was still lower than those associated with other in-vehicle tasks like tuning the radio on an in-vehicle infotainment system (Kim \& Son, 2011). Drivers reported that the headway evaluation system was easy and appealing to use but they were ambivalent about continuing to use it in the future. However, potential applications for the current system include situations where accurate headway information would be beneficial including driver training initiatives or as a means of retaining situation awareness during the use of adaptive cruise control or collision avoidance systems.

\section{CONCLUSIONS}

Tailgating is a major contributor to rear-end collisions but coaching drivers to maintain a safe headway is impeded by their poor capacity to estimate headway. We aimed to compare headway intervention strategies using a DS600c fixed based driving simulator. Before doing so, we established that the headways that drivers maintained in the simulator aligned with previous investigations that have measured driver headway in real vehicles (e.g. Taieb-Maimon, 2007). We then examined the efficacy of a common coaching technique, the 2 second rule, relative to allowing drivers to select their own safe headway. We demonstrated that fully licensed drivers maintained headways below 2 seconds regardless of instruction type. Thus we sought to develop and to test the efficacy of a headway evaluation system, a device designed to present drivers with objective real-time headway feedback along with positive feedback to promote adherence. The system yielded headways that were greater than those achieved with the 2 second rule and that were more conducive to drivers recognizing and evading potential rear-end collisions. While many drivers agreed that the system would be suitable for training purposes, they were not as receptive to the idea of continuing to use it in the future and thus the reward-based component did not have the desired effect. However, the current system could be fruitful in the context of early driver training or as a tool for preserving situation awareness when using driver assistance systems designed to maintain headway autonomously. 


\section{ACKNOWLEDGEMENTS}

Auto21 (Converging Evidence from Naturalistic, Simulation and Epidemiological Data network) the Canadian Foundation for Innovation, and the Ontario Innovation Trust funded this research through grants to the second author. We would like to thank Ryan Toxopeus for his help in these studies.

\section{REFERENCES}

Kim, M. H., \& Son, J. (2011). On-road assessment of in-vehicle driving workload for older drivers: Design guidelines for intelligent vehicles. International Journal of Automotive Technology, 12 (2) 265-272.

Lee, J. D. (2007). Technology and teen drivers. Journal of Safety Research, 38(2), 203-213.

Michael, P., Leeming, F., \& Dwyer, W. (2000). Headway on urban streets: observational data and an intervention to decrease tailgating. Transportation Research F, 3(2), 55-64.

Panerai, F., Droulez, J., Kelada, J.M., Kemeny, A., Balligand, E., Favre, B. (2001). Speed and safety distance control in truck driving: comparison of simulation and a real-world environment. Proceedings of Driving Simulation Conference: DSC 2000 (pp. 91-107). Paris, France: Renault.

Rudin-Brown, C., \& Parker, H. (2004). Behavioural adaptation to adaptive cruise control (ACC): Implications for preventive strategies. Transportation Research Part F: Traffic Psychology and Behaviour, 7(2), 59-76.

Taieb-Maimon, M. (2007). Learning headway estimation in driving. Human Factors: The Journal of Human Factors and Ergonomic Society, 49(4), 734-744.

Taieb-Maimon, M., \& Shinar, D. (2001). Minimum and comfortable driving headways: Reality versus perception. Human Factors: The Journal of Human Factors and Ergonomics Society, 43(1), 159-172.

Wang, C., \& Song, M. (2009, June). Studying the vehicle headway issue and its impact on the slow-down effect. Retrieved from University of Rhode Island Transportation Center: http://www.uritc.uri.edu/media/finalreportspdf/0001852.pdf.

Wang, J. \& Song, M. (2011). Assessing drivers' tailgating behaviour and the effect of advisory signs in mitigating tailgating. Proceedings of the Sixth International Driving Symposium on Human Factors in Driver Assessment, Training and Vehicle Design, Lake Tahoe, California, USA, July 27-30, 2011 (2011)

Wittmann, M., Kiss, M., Gugg, P., Steffen, A., Fink, M., \& Poppel, E. (2006). Effects of display position of a visual in-vehicle task on simulated driving. Applied Ergonomics, 37(2), 187199. 